\title{
2. LA DELIMITACION DE COMPETENCIAS ENTRE EL ESTADO Y LAS COMUNIDADES AUTONOMAS EN MATERIA ELECTORAL
}

PABLO SANTOLAYA MACHETTI

Universidad Complutense. Madrid 


\section{SUMARIO}

1.-Titulos competenciales del Estado y las Comunidades autonomas. II.-EL sistema de La Ley ORgÁNica del Régimen Electoral General. III.-ANÁLISIS DE dos PRoblemas Concretos. 1. La regulación de las elecciones locales. 2. La regulación del Censo Electoral. VI.-A MODO DE CONCLUSIÓN. 
Revista de Derecho Político, núm. 24, 1987, pp. 31-56

\title{
2. LA DELIMITACION DE COMPETENCIAS ENTRE EL ESTADO Y LAS COMUNIDADES AUTONOMAS EN MATERIA ELECTORAL
}

\author{
POR \\ PABLO SANTOLAYA MACHETTI \\ Universidad Complutense. Madrid
}

El objetivo de esta nota es analizar la delimitación de funciones entre el Estado y las Comunidades Autónomas realizada en la Ley Orgánica del Régimen Electoral General ${ }^{1}$, es decir, desde otro punto de vista, pasar revista a los límites de actuación legítima de las Comunidades en sus competencias electorales a la luz de la normativa electoral general.

El trabajo se divide en tres partes: En la primera de ellas recordaremos brevemente los títulos competenciales que corresponden a cada una de las instancias, como labor previa para la delimitación del campo de discusión y analizaremos las leyes electorales elaboradas, excepto la gallega y la andaluza, con anterioridad a la entrada en vigor de la Ley Orgánica del Régimen Electoral General (en adelante LOREG). En la segunda, expondremos el sistema adoptado por la LOREG para tratar de recoger en forma armónica las competencias de las dos instancias. Por último, pasaremos revista a dos problemas concretos que se plantearon a lo largo de su discusión parlamentaria en la esfera de competencias autonómicas: en primer lugar, el ámbito de aplicación de la Ley, en particular el determinar si las Comunidades Autónomas tienen o no facultades de desarrollo de la legislación que regula las elecciones locales, y en segundo lugar, la elaboración del censo, es decir, concretar cuál es la instancia encargada de establecer la legislación y de actuar administrativamente sobre la materia.

1 Ley Orgánica 5/1985 de 19 de junio. BOE 20 de junio. 


\section{TITULOS COMPETENCIALES DEL ESTADO Y LAS COMUNIDADES AUTONOMAS}

El estudio de este apartado debe comenzar por constatar que el legislador constitucional, tras recoger en la propia Constitución algunos de los elementos más importantes del sistema electoral, impone, en el artículo 81,1 un específico mandato de desarrollo de una Ley Orgánica que regule «el régimen electoral general».

Con ello se remite al legislador estatal la responsabilidad de elaborar una norma para el cumplimiento de tres finalidadades fundamentales: completar el sistema electoral en algunos aspectos no totalmente definidos por el Texto constitucional, crear un marco adecuado de garantías procedimentales del ejercicio del derecho de sufragio activo y pasivo, y, por último, conseguir una unidad básica en todo el Estado en el ejercicio de tales derechos y en la estructuración de todas las instancias representativas que de él se derivan en los diferentes niveles territoriales ${ }^{2}$.

Sin embargo, el alcance concreto de este mandato constitucional ha dado lugar a distintas interpretaciones, tanto en lo referente al significado del término "ley electoral» ${ }^{3}$, como al alcance del adjetivo "general»" que, a su vez, determina el contenido materialmente "orgánico» de la norma.

La interpretación del término "general» ha sido objeto de amplio debate en la doctrina; el problema fundamental es dilucidar si con él nos estamos refiriendo exclusivamente a las normas que regulan un determinado tipo de elecciones, las llamadas "elecciones generales" o, por el contrario, si además de esta regulación, el adjetivo se refiere también al término "ley", es decir que la Ley tendría que contener las "normas generales" que son de aplicación a cualquier proceso electoral que se desarrolle en el Estado, aunque naturalmente teniendo en cuenta la existencia de competencias expresamente reservadas por la Constitución a los Estatutos de Autonomía y normativa regional de desarrollo.

Para dilucidar este problema podemos convenir, en principio, con GALVEZ MONTERO ${ }^{4}$ que el adjetivo aplicado a la Ley significa que debe

2 Vid sobre este.plànteamiento de las finalidades de la Ley mi trabajo "Significado y alcance de la Ley Orgánica del Régimen Electoral General”, en Revista de Estudios Politicos. Próxima aparición en el $n .^{0} 52$.

3 Vid a este respecto la Sentencia del Tribunal Constitucional de 14-VI-83, sobre el Recurso previo contra el Proyecto de Ley Orgánica de Incompatibilidades de Diputados y Senadores, declarado inconstucional por ser ésta una materia necesariamente a regular por la Ley Electoral, y no por ninguna otra ley.

J. Galvez MONTERO, "Comentario al artículo 81 de la Constitución", en O. Alzaga (coordinador): Comentarios a las Leyes políticas. Edersa, Madrid, 1985. Tomo VII, pág. 65. 
contener «el régimen jurídico de las elecciones que revisten carácter general, por celebrarse simultáneamente en todo el Estado y con arreglo a normas comunes". Sin embargo, el problema es precisamente dilucidar cuáles son dichas consultas electorales que deben ser objeto de la Ley Orgánica del Régimen Electoral General.

Existen dos aspectos sobre los que se ha producido un amplio acuerdo en la doctrina:

- Son elecciones generales las que se refieren a instancias representativas del Estado en su conjunto, es decir, las del Congreso, Senado y, en un futuro próximo, las del Parlamento Europeo 5 .

- No lo son las previstas para la elección de los Parlamentos autonómicos, aunque la LOREG puede establecer una normativa básica unitaria y actuar también como norma supletoria.

El problema se centra, por tanto, en el carácter general o no de las elecciones locales. Frente a una numerosa doctrina que les negaba tal característica y, en consecuencia, su rango orgánico y su inclusión en la Ley ${ }^{6}$, el Tribunal Constitucional se pronunció en un sentido radicalmente distinto con ocasión de su Sentencia de 16 de mayo de 1983, sobre el Recurso Previo de Inconstitucionalidad contra el Proyecto de Ley Orgánica que modificó la Ley de Elecciones Locales.

que:

En dicha sentencia el Alto Tribunal establece de forma terminante

"El régimen electoral general está compuesto por las normas electorales válidas para la generalidad de las instituciones representativas del Estado en su conjunto y de las entidades territoriales en que se organiza a tenor del artículo 137 de la Constitución española, salvo las excepciones establecidas en la Constitución o en los Estatutos».

5 Sobre las elecciones al Parlamento Europeo vid mi trabajo «El sistema electoral de las elecciones al Parlamento Europeo, un análisis comparadow. En la obra colectiva coordinada por L. AGUIAR DE LUQUE, Implicaciones constitucionales y políticas del Ingreso de España en la CEE y su incidencia en las Comunidades Autónomas. (Actas del V Congreso de la Asociación Española de Ciencia Política y Derecho Constitucional. Bilbao/San Sebastián, octubre 1985). Editadas por el Instituto Vasco de Administración Pública, Oñati, 1986. Págs. 369 y 56. En el libro se pueden encontrar también sobre el tema los trabajos de SuBlrats, Aguilo lucía, Cavero lataillade y Molins López-Rodo.

6 Vid, entre otros, los trabajos de J. Jiménez de CISneros, Ley Electoral. Presidencia del Gobierno. Madrid, 1983 y J. M. ValLES, "Derecho electoral y Ley orgánica», en Revista de Derecho Político. UNED, $n .^{\circ} 18 / 19,1983$. Págs. 123 y ss. 
El Estado tiene, pues, capacidad para introducir como contenido de la Ley Electoral, y con carácter exhaustivo, las normas referentes a todos los procesos electorales "no excepcionados" por la Constitución o los Estatutos de Autonomía; es decir, en primer lugar las de las elecciones al Congreso de Diputados y al Senado, pero también las relativas a las Corporaciones locales y, en un futuro inmediato, las del Parlamento Europeo.

Pero además, el Estado tiene también competencia para regular, mediante esa Ley Orgánica, «lo primario y nuclear de todo proceso electoral", es decir, también de aquellos que correspondan a la acción normativa de las Comunidades Autónomas.

Esta competencia implícita en el enunciado "Ley Electoral General» del artículo 81,1 de la Constitución, se refuerza con el enunciado del $149,1,1$, que le obliga a regular las condiciones básicas que garanticen la igualdad de todos los españoles en el ejercicio de los derechos fundamentales, en este caso el del sufragio, e incluso por el artículo 9,2 que obliga a los poderes públicos a promover de forma positiva la libertad y la igualdad del individuo y los grupos sociales y que, según la doctrina, podría facultar al Estado a intervenir en la regulación electoral de las Comunidades Autónomas para garantizar estos principios, incluso en aquellos aspectos que entran dentro de su ámbito de decisión exclusiva ?

Sólo a partir de estos datos pueden enmarcarse las competencias de las Comunidades Autónomas en la materia, que consistirían, en una primera aproximación, en fijar las "excepciones" al régimen general, en la medida que lo tengan reconocido en la Constitución y en los Estatutos, de forma que en esa actividad están sometidas a bases o principios generales aplicables a toda elección por sufragio universal directo, e incluso, a la eventual y extraordinaria intervención del Estado en defensa de los principios constitucionales.

Así delimitadas, el fundamento constitucional de las competencias de las Comunidades Autónomas en materia electoral se encuentra en el artículo 148,1,1, de la Constitución que establece la facultad de "organización de sus instituciones de autogobierno", y en el 152,1 en el que se prevé, para las Comunidades del 151, la existencia de una «Asamblea legislativa elegida por sufragio universal directo con arreglo a un sistema que asegure, además, la representación de las diferentes zonas del territorio».

7 Vid, en este sentido, el sugerente trabajo de G. Trujillo, «El Estado y las Comunidades Autónomas ante la exigencia constitucional del voto igual", en Revista española de Derecho Constitucional, n. ${ }^{\circ}$. CEC, 1981, en el que realiza atinadas consideraciones sobre el valor del principio de igualdad del sufragio, su posible vulneración por la normativa estatutaria de algunas Comunidades (en especial la Vasca) y la posibilidad de intervención del Estado en casos extremos. 
Por tanto, el primer dato a tener en cuenta es que la normativa electoral de las Comunidades Autónomas ha de estar dirigida única y exclusivamente a regular los procedimientos electorales para la elección de los miembros de los Parlamentos autonómicos, dejando al margen el supuesto especial del Estatuto vasco en relación a los Territorios históricos, competencia autonómica en virtud de la Disposición Adicional $1 .^{a}$ de la Constitución, problema al que nos referiremos más adelante.

Los Estatutos de Autonomía han orientado su regulación en este sentido, $y$ en todos ellos se recogen con mayor o menor detalle una serie de aspectos nucleares de la legislación electoral y se remite el resto de la normativa a su respectiva Ley Electoral, Ley que, por ejemplo, el artículo 11,5 del Estatuto Gallego establece que ha de contener "Los plazos y regular el procedimiento para la elección de los miembros del Parlamento Regional, fijando su número entre 70 y 80 , y las causas de inelegibilidad e incompatibilidad que afecten a los puestos o cargos que se desempeñen dentro del ámbito territorial de la Comunidad». Es decir, que las leyes electorales de las Comunidades Autónomas están orientadas a marcar las peculiaridades de la elección de los órganos legislativos de autogobierno, y en este sentido pueden ciertamente marcar algunas condiciones para el ejercicio del sufragio activo, como es, por ejemplo, la lógica exigencia de ostentar la condición política de ciudadano de la región, que coincide con la nacionalidad española y la residencia administrativa ${ }^{8}$, pueden, con mayor claridad y amplitud, actuar sobre el sufragio pasivo, fijando causas específicamente regionales de inelegibilidad. Pueden, y ésta es, sin duda, su competencia de más amplitud, establecer su propio procedimiento y sistema electoral, siempre respetando la fórmula proporcional impuesta por el 151,2 de la Constitución, su forma de expresión del voto, fijar la circunscripción electoral, que puede o no coincidir con la provincia ${ }^{9}$, siempre que se respete la exigencia de proporcionalidad de las distintas partes del territorio, y, por último, sin ningún ánimo exhaustivo, pueden fijar su propio sistema de incompatibilidades para los elegidos, todo ello, naturalmente, sin que su actuación legislativa provoque una vulneración de la igualdad básica de todos los españoles en el ejercicio de su derecho de sufragio, impuesta en el artículo 149,1,1, vulneración que habilitaría una intervención estatal.

Este marco teórico, hasta el momento, solamente ha sido desarrollado por cuatro Comunidades Autónomas: nos referimos, en concreto, a

B Así, por ejemplo, la Ley de Elecciones al Parlamento Gallego, de 19 de julio de 1985, establece en su artículo 2,1 «son electores los que, ostentando la condición de gallego conforme al artículo $3 .^{\circ}$ del Estatuto de Autonomía, sean mayores de edad y gocen del derecho de sufragio activo".

9 Así, por poner dos ejemplos de Comunidades Autónomas uniprovinciales, mientras la Disposición Transitoria 1. ${ }^{2}$ a. del Estatuto asturiano divide la provincia de Oviedo en tres circunscripciones electorales, oriental, occidental y central, sin embargo, el artículo 18,2 del riojano establece una única circunscripción. 
la Ley Vasca de 25-XI-83, la Catalana de 5-III-84, y ya con posterioridad a la entrada en vigor de la LOREG, la Gallega de 13-VIII-85 y la Andaluza de 2-1-86, aunque es de imaginar que el conjunto de las Comunidades Autónomas elaboren sus Leyes electorales antes de las próximas elecciones regionales en junio de 1987.

Vamos a analizar brevemente el contenido de estas normas ya vigentes:

La Ley Vasca ${ }^{10}$ consiste fundamentalmente en una adaptación del Decreto-Ley del 77 al ámbito propio de la Comunidad Autónoma.

Este carácter está, incluso, expresamente recogido en la exposición de motivos de la norma, que, partiendo del presupuesto -en realidad dudoso con la amplitud con la que es formulado - de que "La Comunidad Autónoma puede regular, como entienda oportuno y conveniente (sic) la organización de los procesos electorales» y de la concepción -aún mucho más discutible - de la no supletoriedad del Derecho estatal, puesto que «en materia electoral no existe norma supletoria por no darse identidad de objeto en sentido estricto, ya que cada norma electoral regula diferentes procesos electorales", sin embargo admite su coincidencia sustancial con el Derecho estatal, y ello porque:

\begin{abstract}
«Es preciso reconocer que la introducción de excesivas modificaciones en las normas que hasta el presente han regulado los procesos electorales... o el establecimiento de un sistema que no se adecua al que en el futuro pueda desarrollarse... puede generar un factor de inseguridad jurídica... (por lo que) el mantener procedimientos homogéneos introduce un factor de confianza y de seguridad subjetiva, componentes indispensables de la seguridad juridica no siempre valorados suficientementem.
\end{abstract}

Así pues, la ley vasca se limita a adecuar la normativa estatal al ámbito territorial de la Comunidad y a otorgar la realización de todas las operaciones administrativas que rodean una elección a órganos de la Comunidad Autónoma, incluyendo el sistema de Juntas Electorales, que según esta Ley culmina en una Junta de Comunidad Autónoma, extremo éste que hay que entender modificado por la LOREG que reserva competencias a la Junta Electoral Central también para las elecciones autonómicas.

A pesar de ello, la Ley contiene dos aspectos concretos en los que el Gobierno de la nación entendió que se vulneraban competencias estatales, y fue por ello impugnada ante el Tribunal Constitucional con fe-

10 Ley 28/83 de 25, XI, de Elecciones al Parlamento Vasco. BOPV, n. ${ }^{\circ} 182$, de 10-XII, 83. 
cha 26-III-84, sin que hasta el momento se haya producido sentencia; la regulación del Censo electoral como competencia de la Comunidad, tema en el que nos detendremos más adelante, y el hecho de que la disposición Adicional $1 .^{a}$ de la Ley introducía en el Concierto económico el coste de las elecciones, es decir, que hacía unilateralmente sufragar las elecciones vascas al Estado, introduciendo su coste en el concierto ${ }^{11}$.

La Ley Catalana ${ }^{12}$ es una regulación provisional, en principio únicamente para las elecciones de 1984, contenida en una curiosísima Ley de artítulo único, que se limita a afirmar la aplicación, en su totalidad, del Decreto-Ley del 77 , pero entendiendo que las competencias atribuidas al Gobierno del Estado y a sus autoridades son asignadas a órganos de la Generalidad en todas las materias que no son competencia exclusiva del Estado, y, además, que las referencias contenidas en el Decreto-Ley al «Boletín Oficial del Estado» lo son al «Diario Oficial de la Generalidad».

Por su parte, tanto la Ley Gallega como la Andaluza ${ }^{13}$ han sido redactadas tomando como modelo, a veces literal, la LOREG sobre la que no se establece novedad alguna digna de consideración, en realidad, a estos efectos y materialmente hablando, no suponen textos muy distintos al de la Ley Catalana que acabamos de exponer.

En definitiva, podemos adelantar como conclusión provisional que las Comunidades Autónomas no han agotado, en modo alguno, el campo posible de su competencia en materia electoral. Este dato es particularmente significativo en los aspectos medulares de la normativa electoral, aquellas materias más altamente políticas de los sistemas electorales; el sistema y la fórmula electoral, la circunscripción electoral y la forma de expresión del voto.

Pues bien, en ninguno de estos aspectos sustanciales a toda normativa electoral se ha introducido novedad alguna digna de mención, se ha reproducido, en todo caso, la normativa estatal, renunciando, por ejemplo, a introducir una fórmula proporcional de resto mayor, que, como es sabido, aumenta la proporcionalidad del sistema en su conjunto, o bien una mayor libertad para el elector mediante algún tipo de voto preferencial, aspectos más criticados del sistema estatal.

11 La Ley del Concierto Económico 12/83 parece violada en dos sentidos, en primer lugar, en cuanto que decide unilateralmente su inclusión en él, ya que el cupo se ha de fijar por una Comisión paritaria. Diputaciones forales/Gobierno Vasco y Administración del Estado, según el artículo 49, y, en segundo lugar, porque se trata de un tipo de gastos que no se pueden incluir en el concierto, al no haberse producido ninguna transferencia en la materia.

12 Ley $5 / 1984$ de 5 de mayo, por la que se adapta la normativa general electoral para las elecciones al Parlamento de Cataluña. Diario Oficial de la Generalidad, n..$^{\circ} 414$, de 7-III-1984.

13 Respectivamente Ley 13 de agosto de 1985, de Elecciones al Parlamento de Galicia (BOE 16-X,1985) y Ley 1/86, de 2 de enero, Electoral de Andalucía (BOE 1-Il-1986). 
Ha habido por tanto una renuncia expresa por parte de las Comunidades Autónomas a modificar en sus líneas esenciales el sistema inaugurado por el Decreto-Ley del 77 y recogido en la LOREG, probablemente por los motivos declarados explícitamente en la exposición de motivos de la Ley Vasca a la que ya nos hemos referido, aunque también conviene señalar que en algunos casos, como en Andalucía, la renuncia estaba ya en el propio Estatuto ${ }^{14}$.

\section{EL SISTEMA DE LA LEY ORGANICA DEL REGIMEN ELECTORAL GENERAL}

El articulado de la LOREG se abre con un Título Preliminar, que consta de un único artículo en el que se señala el ámbito de aplicación de la norma en los siguientes términos:

“1) La presente Ley es de aplicación: a) A las elecciones de Diputados y Senadores a Cortes Generales sin perjuicio de lo dispuesto en los Estatutos de Autonomía para la designación de los Senadores previstos en el artículo 69,5 de la Constitución. b) A las elecciones de los miembros de las corporaciones locales. 2) Asimismo, en los términos que establece la Disposición Adicional Primera de la presente Ley es de aplicación a las elecciones a las Asambleas de las Comunidades Autónomas, y tiene carácter supletorio de la legislación autonómica en la materia».

Asi pues, a tenor de este artículo, el «régimen electoral general» contendrá normas aplicables, aunque de forma distinta, a todos los procesos electorales de las instancias político-representativas, tanto del Estado en su conjunto como de las entidades territoriales en que se articula, a tenor del artículo 137 de la Constitución.

Se excluye únicamente de este marco la designación de los Senadores representantes de las Comunidades Autónomas, que el artícu10 69,5 de la Constitución remite a los Estatutos de las Comunidades Autónomas, con un amplio margen de libertad a la normativa autonómica, con los únicos límites de que se trate de una designación parlamentaria y de que se asegure la adecuada representación proporcional entre los

14 Es muy significativo, en este sentido, el articulo 28,2 del Estatuto andaluz: «La elección se verificará atendiendo a criterios de representación proporcional. Se utilizará para ello el mismo procedimiento que rija para las elecciones al Congreso de Diputados». 
grupos políticos de la Asamblea ${ }^{15}$. Esta exclusión se debe precisamente a que no nos encontramos ante una elección sino ante una designación que proviene, además, de órganos regionales.

Al margen de este supuesto, como ya hemos señalado, la LOREG contiene normativa aplicable a todos los procesos electorales que se celebren en nuestro país, pero lo hace de forma distinta, según el proceso concreto de que se trate.

a) Con respecto a las Cortes Generales y a las Corporaciones locales, contiene la normativa directamente aplicable a ambos tipos de elecciones, de forma marcadamente globalizadora y reglamentista, al haberse incluido en el texto gran cantidad de materias que en el sistema anterior tenían rango meramente reglamentario. De esta forma y sin perjuicio de que, por supuesto, la materia necesite en alguno de sus aspectos desarrollo reglamentario ${ }^{16}$, se ha optado por hacer aprobar por las Cortes Generales, sustrayéndolo del campo de actuación del Gobierno, prácticamente toda la regulación que configura el procedimiento electoral.

Surge ante este hecho un problema de forma inmediata; ¿significa que toda la materia regulada en la norma es orgánica? ${ }^{17}$. La respuesta ha de ser contundentemente negativa, el detallismo y reglamentarismo de la norma impiden predicar ese carácter de numerosos de sus artículos. Por el contrario, hay que concluir que el legislador ha hecho un uso intensísimo de la técnica de las "materias conexas", tal como fue definida en la Sentencia del Tribunal Constitucional de 13-II-81 ${ }^{18}$. Este hecho provoca el efecto de "congelación de rango" característico de las Leyes or-

15 Vid, en este sentido, la importante STC de 18-XII-1981, que declara la constitucionalidad de la Ley del Parlamento Vasco 4/81, de 18 de marzo, sobre “Designación de Senadores representantes de Euskadi». Vid, sobre la misma, mi libro Descentralización y cooperación. Instituto de Estudios de Administración Local. Madrid, 1984. En especial, páginas 482-497.

16 Y así se ha producido con posterioridad a la entrada en vigor de la LOREG, por ejemplo, por el Real Decreto 1732/85, de 24 de septiembre «por el que se regulan las condiciones de los locales y las características oficiales de los elementos materiales a utilizar en los procesos electorales" y por el 1733, de la misma fecha, "sobre solicitud del voto por correo en caso de enfermedad o incapacidad que impida formularla personalmente". ya citado.

17 . A este problema me he referido en "Significado y alcance de la Ley Orgánica..."

${ }_{18}$ Me refiero a la Sentencia sobre el Estatuto de Centros Escolares, en la que, tras definir un concepto al mismo tiempo material y formal de Ley Orgánica, afirma, sin embargo, que «el legislador, al elaborar una Ley Orgánica podrá sentirse inclinado a introducir en ella el tratamiento de cuestiones regulables por vía reglamentaria, pero que, en atención a razones de conexión temática, de sistematicidad 0 . de buena política legislativa considere oportuno incluir junto a las materias estrictamente reservadas a la Ley Orgánica». Doctrina que no ha dejado de provocar algunas críticas. Vid, por ejemplo, T. R. FERNANDEZ "Las Leyes orgánicas", en Las leyes orgánicas y el bloque de constitucionalidad". Cuadernos Civitas, Madrid, 1981, en especial, págs. 87 y ss. 
gánicas, y que en principio se predica en todo el Texto, y en concreto con respecto a los artículos recogidos en el Capítulo I, que es el destinado a las «Disposiciones Comunes para las elecciones por sufragio universal directo", que son los que interesan a la hora de determinar las competencias de las Comunidades Autónomas. La propia Ley Orgánica se encarga de establecer por via indirecta qué artículos no son materialmente orgánicos al no declararlos en el párrafo $2 .^{\circ}$ de la Disposición Adicional $1 .{ }^{\mathrm{a}}$, necesariamente aplicables a las elecciones regionales, problema éste al que nos referiremos inmediatamente.

b) Con respecto a las elecciones de las Asambles legislativas de las Comunidades Autónomas, la ley es aplicable "en los términos que establece la Disposición Adicional $1 .^{\mathbf{a}}$, en la que es necesario detenerse con cierto detalle.

El párrafo $1 .^{\circ}$ de la referida Disposición Adicional se abre con el reconocimiento general de las competencias de las Comunidades Autónomas, utilizando para ello una de las célebres causas "sin perjuicio" que tan acertadamente han sido criticadas por la Doctrina y que parecen caracterizar el desarrollo de nuestra legislación estatal y autonómica ${ }^{19}$ : "Lo dispuesto en esta Ley se entiende sin perjuicio del ejercicio de las competencias reconocidas, dentro del respeto a la Constitución y a la presente Ley Orgánica, a las Comunidades Autónomas por sus respectivos Estatutos".

Con esta cláusula se trata de definir el «bloque de constitucionalidad» aplicable a la materia; las Comunidades Autónomas están, por supuesto, sujetas a la Constitución en el desarrollo de su competencia electoral, pero también a la Ley Electoral General en la medida en que respete sus ámbitos competenciales estatutarios.

El párrafo segundo es una especificación de los artículos que son de aplicación directa a las elecciones regionales, y que, por lo tanto, no pueden ser objeto de normativa por parte de las Comunidades Autónomas por tratarse de materias reservadas por la Constitución al Estado, contenido propio de la LOREG; es decir, por ser materialmente orgánicos o requerir un tratamiento idéntico en todo el territorio nacional para garantizar la igualdad en el ejercicio del derecho fundamental de sufragio.

Entre estas materias, todas ellas recogidas en el articulado del Título I de la Ley, por la propia estructura de la norma, se pueden señalar las siguientes:

19 Vid sobre el tema el interesantismo trabajo de S. MuÑoz MACHADo «Deformaciones del ordenamiento y aplicación de leyes en el nuevo sistema constitucional», en Documentación administrativa, n. 200,1984 ; págs. 43 y ss. 
a) La regulación del Derecho de sufragio activo y pasivo, contenida en los artículos 2 a 7 de la Ley, incluyendo cláusulas generales de inelegibilidad, que pueden ser completadas, pero no modificadas, para las elecciones autonómicas por la normativa regional, de la misma manera que las disposiciones especiales de la Ley lo hacen para cada tipo de elecciones que regulan en su totalidad ${ }^{20}$. El carácter fundamentalmente unitario de esta regulación está plenamente justificado por la sustancial igualdad que la Constitución garantiza a todos los ciudadanos del Estado en el ejercicio de los derechos fundamentales que quebraria si cada región pudiera regular la materia de forma absolutamente diferente.

b) La existencia de una Administración electoral fuertemente judicializada, que tiene por objeto, según el artículo $8 .^{\circ}$ "garantizar la transparencia y objetividad del proceso electoral y el principio de igualdad».

Con este fin se fija en la ley de composición y funcionamiento de los tres niveles de Juntas electorales que se crean y su necesaria actuación en todo tipo de elecciones. Son las Juntas Central, Provinciales y Locales, $y$, junto a ellas, se arbitra la posibilidad de que la legislación autonómica cree la Junta Electoral de Comunidad Autónoma, como instancia suprema para las elecciones regionales, pero bien entendido que esto no significa merma de las atribuciones reservadas a la Junta Electoral Central. Se trata, además, de un órgano facultativo, cuya existencia, composición y funciones han de ser reguladas por la Ley de cada una de las Comunidades Autónomas, como así, efectivamente, lo han hecho tanto la Ley Gallega como la Andaluza de forma absolutamente paralela a la Junta Electoral Central, en cuanto a su carácter permanente, composición y sistema de nombramiento, funciones... ${ }^{21}$.

c) La regulación de las Mesas y Secciones electorales, asi como la existencia de un Presidente y dos Vocales en cada mesa para la realización de las operaciones electorales (arts. 23-28).

20 Así, por ejemplo, la Ley gallega señala en su artículo $4 .^{\circ}$ como ineligibles a aquellos que lo son según la legislación general, y, además, una serie de autoridades gallegas y estatales.

21 Vid los artículos 13 al 18 de la Ley gallega y 7 al 13 de la andaluza. Es de esperar que el sistema se generalice, lo que en alguno de sus puntos es criticable, por ejemplo, en su carácter de órgano permanente, ya que tal nota tiene justificación en la Junta Electoral Central, en cuanto actúa en todos los procesos electorales en un calendario especialmente saturado como es el de nuestro país, pero resulta improcedente en un órgano que, sólo se va a ocupar, con carácter general, de unas elecciones regionales cada cuatro años, en especial teniendo en cuenta la prohibición de disolución de las Asambleas regionales contenida en buen número de Estatutos. Sin embargo creemos que el mecanismo de «imitación institucional» que parece una constante en nuestras Comunidades Autónomas actuará también en este supuesto, incluso en Comunidades uniprovinciales con circunscripción única, donde realmente no tendría ninguna justificación. 
d) La configuración del Censo como un documento único en todo el territorio nacional para todo tipo de elecciones, elaborado por un órgano estatal de nueva creación: la Oficina del Censo Electoral y sus Delegaciones provinciales. Se trata de un órgano administrativo, encuadrado en el Instituto Nacional de Estadística, pero que (art. 29) “ejerce sus competencias bajo la dirección y supervisión de la Junta Electoral Central's.

Esta opción por la "estatalidad» del censo a todos los niveles, consecuencia directa de la importancia trascendental del instrumento censal para el ejercicio del derecho de sufragio, fue uno de los puntos más problemáticos de la tramitación de la Ley, ya que, como veremos más adelante, algunas Comunidades Autónomas, particularmente la vasca, reclamaban, en virtud de su atribución en «estadística para sus propios fines y competencias", la elaboración de su propio censo electoral al menos en sus aspectos administrativos.

e) Ciertas condiciones para la presentación de candidatos, como por ejemplo la delimitación de los sujetos que pueden presentar candidatos o listas de candidatos (art. 44) o algunas normas sobre el contenido del escrito de presentación (art. 45) como garantía de la igualdad básica en el ejercicio del derecho de sufragio pasivo.

f) Algunas normas mínimas generales relativas a la campaña electoral, como por ejemplo la prohibición de discriminación entre candidaturas a efectos de publicidad (art. 58) o la fijación del número de votos obtenidos en las anteriores elecciones equivalentes como criterio para la distribución de los espacios de propaganda electoral en los medios de comunicación de titularidad pública (art. 61).

g) Ciertas condiciones mínimas para el ejercicio del Derecho de voto en estricta aplicación de un mandato constitucional (por ejemplo, el carácter secreto) o directamente derivado de ella (por ejemplo, el carácter público del escrutinio) (art. 95.2).

h) Toda la materia referida al contencioso-electoral (arts. 109-

i) El capítulo de Delitos electorales y el procedimiento judicial para su sanción.

Queda, por tanto, a las Comunidades Autónomas un amplio campo de actuación en sus Leyes electorales. Sin ánimo exhaustivo podemos señalar, en primer lugar, todas las disposiciones relativas al sistema electoral, auténtico núcleo político de cualquier norma electoral, como el de 
las circunscripciones electorales (que pueden o no coincidir con las utilizadas para las elecciones generales), la fórmula electoral (que puede ser cualquiera que tenga carácter proporcional), la fórmula de expresión de voto (virtualmente cualquiera, incluidas las listas abiertas) y, además, un importante elenco en materia de procedimiento, como la existencia o no de Junta de Comunidad autónoma, sus funciones y composición; de la figura de los representantes; de los apoderados; de los interventores; los procedimientos de constitución y funcionamiento de las Mesas (salvo lo señalado para la distribución en Mesas y Secciones en los artículos 23 al 28); prácticamente toda la materia de escrutinio en las Mesas y de escrutinio general... y, por último, - last but not least - decidir sobre la existencia o no de subvención electoral a los grupos políticos, establecer los criterios, las cuantías y sus propios controles de gastos y subvenciones.

Hay que señalar un último dato significativo con respecto a este párrafo $2 .^{\circ}$ que estamos analizando. Como hemos visto, en él se señalan aquellas materias que por estar reservadas por la Constitución al Estado se aplican también a las elecciones convocadas por las Comunidades Autónomas. Pues bien, este texto surgido del Parlamento es, exactamente, el negativo del que fue en su día enviado por el Gobierno. En efecto, el texto gubernamental del antiguo artículo 207 afirmaba:

«En el ámbito de las facultades y competencias determinadas por sus respectivos Estatutos, las Comunidades Autónomas podrán modificar o sustituir los siguientes articulos de esta Ley...

e incluía el listado exactamente inverso al actual.

Es decir, que siguiendo el esquema inaugurado por el Estatuto de Centros Escolares, declarado constitucional por la Sentencia de 13-II-81, el proyecto marcaba positivamente el ámbito de actuación de las Comunidades Autónomas, y se señalaban un elenco concreto de artículos que las Comunidades podían «modificar o sustituir»; frente a ello, el texto final ha acabado por señalar las competencias estatales, lo inmodificable para la actividad legislativa de las Comunidades Autónomas.

Este "cambio de perspectiva", todo lo importante que se quiera desde el punto de vista de la filosofía autonómica de la Ley, en cuanto parece suponer el reconocimiento de una competencia «general» de las Comunidades Autónomas - aunque limitada por la propia Ley-, sin embargo desde la materialidad de las competencias, desde la amplitud de poderes de una $u$ otra instancia carece en realidad de toda trascendencia.

La modificación fue introducida en el Pleno del Congreso a instancias de Minoría Catalana, y es curioso señalar que produjo un cambio sustancial en la postura de ese grupo, que pasó de anunciar un recurso 
de inconstitucional al proyecto, a votar a favor de él en la Cámara baja y a no mantener ni una sola enmienda para su tramitación en el Senado. parte:

El párrafo tercero de la Disposición Adicional establece por su

\begin{abstract}
"Los restantes artículos del Título I de esta Ley tienen carácter supletorio de la regulación que en su caso aprueben las Comunidades Autónomas, siendo de aplicación en las elecciones a sus Asambleas legislativas en el supuesto de que las mismas no regulen sobre ellos».
\end{abstract}

Este apartado constituye una reiteración innecesaria del artículo $1 .^{\circ}$ que señala el carácter supletorio de la Ley, especificando tal carácter otorgado al derecho estatal por el artículo 149,3 de la Constitución.

Pero además de ser innecesario, introduce un cierto factor de confusión al señalar únicamente el carácter supletorio del Título I y no del conjunto de la Ley. Ahora bien, por la propia estructura de la norma existen numerosas disposiciones del Título I que son meras remisiones a la regulación contenida en los restantes Títulos para cada tipo de elecciones, sin que en sí mismos puedan ser aplicados más que completándolos con la disposición pertinente, referida a las elecciones al Congreso y Senado o a las locales.

Para explicar el problema vamos a suponer que una determinada Comunidad Autónoma no regula cuál es la Junta Electoral competente para aprobar el modelo oficial de papeletas. En este supuesto concreto, la supletoriedad del Título I, en este caso del artículo 70,1, no resuelve el problema en absoluto, ya que dicho artículo se limita a remitir a las disposiciones especiales de la Ley para determinar la Junta competente. Junta que será respectivamente la provincial (art. 172,1) o la de zona $(189,1)$ según el tipo de elecciones.

Al margen del supuesto concreto, lo que hay que señalar es el dato de la inadecuada referencia a la supletoriedad del Título I; es, por el contrario, el conjunto de la Ley el que reviste esa característica y, en especial, por la naturaleza de las elecciones autonómicas, en las que se eligen órganos dotados de la facultad de definir indirizzo político, el título dedicado a las elecciones a Cortes Generales.

En este sentido, tanto la Ley electoral gallega como la andaluza han introducido sendas disposiciones finales, en las que se recalca la supletoriedad del Título de las Elecciones Generales, aunque con una fórmula, por cierto, no excesivamente feliz. Así la Disposición Final primera del texto andaluz señala: 
“En todo lo no previsto en esta Ley serán de aplicación las normas vigentes para las elecciones al Congreso de los Diputados de las Cortes Generales, con las adaptaciones y modificaciones derivadas del carácter y ámbito de la consulta electoral al Parlamento de Andalucía y, en este sentido, se entiende que las competencias atribuidas al Estado y a sus órganos y Autoridades se asignan a las correspondientes de la Comunidad Autónoma respecto de las materias que no son competencia exclusiva de aquél».

Fórmula ésta en la que no se puede dejar de observar la influencia de la Ley Electoral catalana que hemos comentado con anterioridad.

Por su parte, el párrafo cuarto de la Disposición Adicional refuerza la idea de que la actuación normativa de las Comunidades Autónomas sólo se puede referir a las elecciones a sus Asambleas legislativas (dejando al margen el supuesto especial del Pais Vasco al que nos referiremos más adelante), sin que en virtud de ninguna de sus competencias, y en particular de las que les pertenecen en régimen local, puedan recabar la regulación de otro tipo de elecciones.

Así, este apartado señala que el contenido de los Títulos Segundo (Elecciones de Diputados y Senadores), Tercero (municipales), Cuarto (Cabildos insulares canarios) y Quinto (Diputaciones provinciales) no pueden ser modificados o sustituidos por la legislación de las Comunidades Autónomas.

Por último, la Disposición Adicional se cierra con un párrafo quinto que establece unos criterios interpretativos destinados a hacer directamente aplicable la Ley a las elecciones para las Asambleas de las Comunidades Autónomas, en el supuesto de que las mismas no legislen sobre determinados temas.

Así pues, el párrafo $A$ prevé la sustitución de organismos estatales por autoridades autonómicas, a los efectos de asegurar la disponibilidad de papeletas electorales y de designación de una persona para recoger información oficial sobre los escrutinios en las mesas, el $B$ ) especifica que a los efectos de distribución de espacios gratuitos, la mención al territorio nacional del artículo 64,1 se sustituye por la del territorio de la Comunidad Autónoma de que se trate, y, por último, el $C$ ), de mucha mayor trascendencia, responsabiliza a las Comunidades Autónomas para financiar y controlar los gastos electorales en el supuesto de que no legislen sobre el tema.

En general, el sistema de distribución de competencias realizado por la LOREG ha sido perfectamente aceptado por el conjunto de los partidos políticos, incluidos el Partido Nacionalista Vasco y Convergencia i Unió, que como es sabido ha apoyado esta Ley a lo largo de su tramitación parlamentaria. Se puede, por lo tanto, afirmar, con carácter general, que el aspecto autonómico de la Ley no ha resultado conflictivo. Sin em- 
bargo, se plantearon dos problemas a lo largo de la tramitación parlamentaria que merecen una cierta reflexión, y a lo que vamos a dedicar el último apartado de este trabajo; en concreto los referidos al carácter de la normativa de las elecciones locales y el de la regulación del Censo electoral.

\section{ANALISIS DE DOS PROBLEMAS CONCRETOS}

\section{La regulación de las elecciones locales}

Dentro de este apartado conviene distinguir, por afectar a problemas distintos dentro de la materia, las posturas mantenidas durante la tramitación de la LOREG por tres grupos políticos distintos: el partido Comunista, la Minoría Catalana y el Grupo Parlamentario Vasco.

\section{Partido Comunista}

La posición del Partido Comunista, a este respecto, se plasma, entre otras, en la enmienda núm. 364, presentada en el Congreso de los Diputados por el señor Carrillo, al artículo $1 .^{\circ}$ de la Ley, y que pretende que la norma sea aplicada "a las elecciones de los miembros de las corporaciones locales, sin perjuicio del desarrollo legislativo de las competencias que sobre régimen local atribuyen los Estatutos de Autonomía".

Es decir, que para el Partido Comunista, el Estado se debería de limitar a emanar unas normas básicas en la materia de elecciones locales, normas que serian complementadas mediante las oportunas leyes de desarrollo dictadas por las Comunidades Autónomas, en virtud de sus competencias de régimen local.

Desde el punto de vista formal, esta postura presenta dos problemas fundamentales:

a) En primer lugar, trata de basar las competencias autonómicas para el desarrollo de la normativa atinente a las elecciones locales en la materia de "régimen local», en manifiesta vulneración de la doctrina sentada por el Tribunal Constitucional en su Sentencia de 16 de mayo de 1983, que expresamente califica esta argumentación de "criterio inadmisible... por apoyarse en una interpretación ciertamente forzada y extensiva... frente a la cual se alza la necesidad de atribuir todo el valor y al- 
cance que merece la expresión "régimen electoral general" incluida en el artículo 81,1 de la Constitución».

b) En segundo lugar, esta postura es intrínsecamente incoherente; se enmienda el artículo $1 .^{\circ}$ de la Ley en el sentido señalado, pero no las disposiciones relativas a las elecciones locales en el cuerpo de la Ley, lo que supone que, de haber prosperado la enmienda, todo el sistema previsto en la Ley para las elecciones locales hubiera pasado a ser "básico", pero como lo alli regulado es un sistema completo sumamente procedimental y detallista, no hubiera quedado campo alguno para el «desarrollo» por parte de las Comunidades Autónomas, hubiera supuesto un cambio puramente semántico privado de todo contenido material.

En cuanto al fondo del problema, y partiendo de la legitimidad jurídica de un sistema unitario de elecciones locales, no cabe tampoco duda alguna sobre la oportunidad política y organizativa de tal solución; no se alcanza a ver las ventajas -en términos de autonomía- que alcanzarían las Comunidades Autónomas si pudieran legislar sobre la materia, y si, por el contrario, los claros inconvenientes de la celebración de las elecciones locales bajo 17 fórmulas organizativas matizadamente distintas.

\section{Minoría Catalana}

Por su parte, la Minoría Catalana dirige sus enmiendas dentro de esta materia a un aspecto distinto: su pretensión se refleja, entre otras, en la enmienda núm. 534 que afirma: «Las Comunidades Autónomas que tengan reconocida una especial forma de organización territorial local podrán acomodar a aquélla las disposiciones de la presente Ley."

En el fondo del problema subyace la aspiración catalana de proceder a una reorganización de su territorio sobre base comarcal, aspiración que tiene reflejo estatutario: (art. 2: «el territorio de Cataluña como Comunidad Autónoma es el de las comarcas comprendidas en las provincias de... art. 5,1: «La Generalidad de Cataluña estructurará su organización territorial en municipios y comarcas...") y que ha sido en cierta forma recogida en la nueva Ley de Bases de Régimen Local, de 2 de abril de 1985, en su Disposición Adicional 4. ${ }^{\text {: }}$ ( «En el supuesto de que... se impidiera (por los Ayuntamientos)... de forma parcial y minoritaria la organización comarcal del conjunto del territorio de la Comunidad Autónoma, la Generalidad de Cataluña, por haber tenido aprobada en el pasado una organización comarcal para la totalidad de su territorio y prever su Estatuto, asimismo, una organización comarcal de caracter general podrá, mediante Ley aprobada por mayoría absoluta de su Asamblea legislativa, acordar la constitución de la comarca o comarcas que resten para extender dicha organización a todo su ámbito territorial»). 
En este contexto, la enmienda, de haber prosperado, hubiera supuesto una habilitación a la Comunidad catalana para utilizar el sistema de elección de las Diputaciones provinciales, pero aplicado, en su ámbito territorial, no sobre base provincial sino comarcal.

En realidad, nos encontramos ante un tema constitucionalmente resuelto por la Sentencia de 28 de julio de 1981, que declaró inconstitucional la Ley catalana de «Transferencia urgente y plena de las Diputaciones catalanas a la Generalidad».

En dicha sentencia, el Tribunal confirma la provincia como una institución básica cuya preservación se juzga necesaria para asegurar los principios constitucionales, elemento arquitectónico indispensable del orden constitucional y como tal protegido con una garantía institucional, indisponible para el legislador estatal y autonómico, que obliga a que sea preservada en términos recognoscibles.

Pues bien, en este sentido parece claro que la vulneración más radical de la garantía institucional de un ente que goza de autonomía para la gestión de sus intereses (art. 137 de la Constitución) y que como tal su gobierno y administración autónoma están recomendados a "Diputaciones y otras corporaciones de carácter representativo" (art. 141,2) es precisamente el privarla de órganos de autogobierno, sin los cuales ningún tipo de autonomía es siquiera concebible. Hay que concluir, por tanto, que la pretensión catalana era contraria a la Constitución.

\section{Grupo Parlamentario Vasco}

La posición del Grupo parlamentario vasco en esta materia viene condicionada por la particular regulación contenida en el Estatuto de Autonomía en relación a las corporaciones locales. Son, en concreto, dos los artículos de dicho Estatuto que merecen una especial consideración:

a) En primer lugar, el artículo 10.3 que establece, entre las competencias exclusivas de la Comunidad Autónoma, la de "Legislación electoral interior que afecte al Parlamento Vasco, Juntas Generales y Diputaciones forales...".

Este artículo, sin paragón en ningún otro Estatuto de Autonomia, supone que las Juntas Generales y Diputaciones provinciales reciben un tratamiento distinto a los órganos de gobierno de las provincias del resto de España, es decir a las Diputaciones provinciales. El fundamento constitucional de esta diferencia se encuentra en la Disposición Adicional Primera, según la cual: «La Constitución ampara y respeta los derechos históricos de los territorios forales" que, en este caso, han sido "actualizados" por el Estatuto de Autonomía, otorgando a la Comunidad del País Vasco la competencia de establecer legislación electoral que afecta a estas instituciones. 
La consecuencia práctica de este fenómeno es, por lo tanto, que las elecciones a las Juntas Generales y Diputaciones Forales del País Vasco van a ser reguladas por Leyes de la Comunidad Autónoma, al contrario de lo que ocurre con las Diputaciones provinciales.

Nos encontramos, por lo tanto, ante una de las "excepciones» al ámbito de la Ley Electoral General, como señala la tantas veces citada Sentencia de 20 de mayo de 1983, y cuyo carácter, también materialmente "excepcional», ha sido puesto de manifiesto por la doctrina ${ }^{22}$.

Pues bien, con tal carácter ha sido recogida en la LOREG, en su artículo 209, cerrando el capítulo III (procedimiento electoral) del Título V (Elecciones a las Diputaciones provinciales), al afirmar: «Lo dispuesto en el presente capítulo se entiende sin perjuicio del respeto a los regímenes especiales, autonómicos y forales».

Este artículo significa que el Parlamento Vasco puede elaborar las normas procedimentales y de sistema electoral que considere oportunas para las elecciones de las Diputaciones forales, pero habrá, sin embargo, de utilizar las relativas al sufragio activo y pasivo establecidas con carácter general para las Diputaciones provinciales.

b) El segundo de los artículos a los que nos referiamos es el 37 del Estatuto Vasco, que establece como competencia exclusiva de los "Territorios históricos", dentro de su ámbito territorial, la referida al «Régimen electoral municipal».

Nos enfrentamos con este tema a un problema extremadamente delicado; del enunciado del Estatuto parece derivarse que las normas relativas a las elecciones municipales van a proceder en el País Vasco de los órganos de autogobierno de los Territorios Históricos, y, además, esta potestad aparece confirmada en el artículo 7,2 de la Ley de Territorios Históricos ${ }^{23}$, que atribuye a los órganos forales la competencia exclusiva en «Régimen electoral y de entidades menores", y en el 7,1 la referente a "las normas electorales... de sus órganos forales". cias.

Se trata, por tanto, de determinar el alcance de dichas competen-

Para ello hay que partir de una afirmación que resulta absolutamente obvia; el régimen electoral de las elecciones locales ha de ser establecido mediante Ley, y no por norma de ningún otro tipo. Esta conclusión se obtiene de la mera lectura de los artículos 140: «los concejales

22 Vid, por ejemplo, E. Garcla de Enterriat. R. Fernández, Curso de Derechó Administrativo, Vol. I, Civitas. Madrid, 4. ${ }^{a}$ ed., 1983, pág. 185.

${ }_{23}$ Ley vasca $27 / 83$ de 25 de noviembre de "Relaciones entre las instituciones comunes de la Comunidad Autónoma y los órganos forales de sus territorios históricos" BOPV de 10-XII-83. 
serán elegidos... en la forma establecida por la Ley». 23,2: «...con los requisitos que señalan las leyes". 53,1: “sólo por Ley podrá regularse...". 86,2 (prohibición de Decreto-ley)...

Esta Ley ha de ser, además, Ley Orgánica, por imperativo del artículo 81,1 de la Constitución, excepto para los casos excluidos en la propia Constitución o en los Estatutos, como son ciertamente las Diputaciones forales Vascas, que pueden ser reguladas por las Comunidades Autónomas, pero respetando en todo caso la reserva legal a la que está sometida la materia. Reserva que, por supuesto, excluye la utilización de cualquier otro instrumento normativo, pero que va más allá, al no ser suficiente, como ha declarado la Sentencia del Tribunal Constitucional de 14 de junio de 1984, - a la que ya nos hemos referido con anterioridadcualquier Ley, sino precisamente la Ley Electoral, al menos en lo que se refiere al núcleo esencial de la materia.

Planteada así la cuestión, surge la pregunta de si esa reserva legal puede ser satisfecha por la actuación normativa de los Territorios Históricos, lo que, a su vez, nos lleva a plantearnos si las normas forales son auténticas leyes que, en su ámbito competencial, son jerárquicamente equiparables a las leyes del Estado y de las Comunidades Autónomas.

La respuesta ha de ser necesariamente negativa, ya que, como ha señalado certeramente TOMAS RAMON FERNÁNDEZ: "No hay ni puede haber ley sin previsión constitucional expresa e inequívoca al respecto. En nuestra Constitución la potestad legislativa está reservada al Estado y a las Comunidades Autónomas... ni directa ni indirectamente cabe, pues, atribuir tal potestad a otro tipo de entes" 24 .

Por ello hay que concluir que, a pesar de la evidente y querida ambigüedad de la Ley de Territorios Históricos, que distingue en su artículo $8 .^{\circ}$ entre una supuesta facultad normativa (caracterizada por la aplicación de las normas forales con preferencia a cualesquiera otras) y una facultad reglamentaria, en realidad, en todo caso, "Los Territorios Históricos pueden y deben ejercer su potestad normativa autónoma a través de los productos normativos subordinados a las leyes que corresponden a aquellas corporaciones territoriales o locales integradas en algún ente territorial superior dotado de soberanía (el Estado) o de autonomía politica (la Comunidad Autónoma)", es decir, a través de normas jurídicas reglamentarias ${ }^{25}$.

Así pues, sus competencias están condicionadas por el doble factor del límite de las de la Comunidad Autónoma, por una parte, y, por otra, por su falta de capacidad legislativa. Es decir, que los Territorios Histó-

24 T. R. Fernandez, Los Derechos historicos de los territorios forales. CEC/Civitas. Madrid, 1985, pág. 142.

${ }_{25}$ J. LEGUinA, «Los territorios históricos vascos: poderes normativos y conflictos de competencias", en Escritos sobre autonomias territoriales. Tecnos, Madrid, 1984, pág. 216. 
ricos no pueden tener ni competencias de las que carece estatutariamente la Comunidad, ni se les puede atribuir el ejercicio de aquellas que precisen del instrumento de la Ley.

En definitiva, y excluida la hipótesis de que las normas forales puedan regular in totum la materia de elecciones locales, hay que concluir que los Territorios Historicos gozarán de una facultad meramente reglamentaria en aquellos contenidos electorales -de ámbito provincialen los que la Comunidad Autónoma posee facultad legislativa, es decir, en lo que se refiere a la elección de Juntas Generales y Diputaciones forales, y no para la Asamblea legislativa (que corresponde a la Comunidad) y, por supuesto, tampoco para las elecciones municipales (que son competencia del Estado) ${ }^{26}$.

Una consecuencia que se deriva de la afirmación anterior es que es la legislación autonómica la que ha de determinar el alcance concreto de esta potestad reglamentaria y no la LOREG.

De este modo, el Parlamento Vasco aprobó, con fecha 7 de mayo de 1983, tres leyes electorales para las Juntas Generales de los tres territorios históricos, en las que resulta manifiestamente claro el auténtico alcance de la pretendida competencia exclusiva de estas instancias; la única competencia que se reconoce a un órgano foral es la facultad del Diputado General de cada Territorio Histórico de convocar las elecciones, pero de forma que «haga coincidir la fecha y plazos de las mismas con las de las elecciones municipales" ${ }^{27}$.

\section{La regulación del Censo Electoral}

El segundo de los problemas que surgieron a lo largo de la tramitación de la LOREG se refiere a la actuación en materia censal. La Ley Electoral establece, en sus artículos 29 y siguientes, un órgano administrativo estatal: la Oficina del Censo Electoral, como el encargado para todo el territorio nacional de las operaciones relacionadas con el Censo Electoral.

Se opta, por tanto, en la Ley por la existencia de un documento censal único atribuido, en cuanto a su elaboración, a un órgano estatal

26 Vid en este sentido E. MENCISIDOR, "Sistema electoral y partidos políticos en el Estatuto de Autonomía del Pais Vasco", en Las Jornadas estudio Estatuto Autonomía del País Vasco. Instituto Vasco de Estudios de Administración Pública. Oñati, 1983. Tomo I, págs. 278-377, en especial 330 y ss.

27 Vid artículos 5,2 de la Ley $2 / 83$ de 7 de mayo, para Guipúzcoa, de la Ley 3 de la misma fecha, para Vizcaya, y de la 4, también de la misma fecha, para Alava. Los tres textos se pueden encontrar en el Boletín de Legislación de las Comunidades Autónomas, n. 2 , Cortes Generales, 1983, págs. 340-343. 
estructurado en delegaciones provinciales y que actúa bajo la dirección y supervisión de la Junta Electoral Central. Se excluye, en consecuencia, toda actividad normativa y ejecutiva de las administraciones autonómicas.

Frente a este planteamiento, tanto el Grupo parlamentario vasco como el catalán, defendieron a lo largo de la tramitación parlamentaria de la Ley la configuración de la Oficina del Censo como un órgano de supervisión y coordinación de la actividad de las administraciones autonómicas en la materia, actividad que tendría su base en las competencias en materias estadísticas y electorales.

El problema ya había causado, como ya hemos señalado, la impugnación de la Ley de Elecciones al Parlamento Vasco, que aún continúa sin ser resuelta por el Tribunal Constitucional.

Dicha impugnación se dirigía fundamentalmente al artículo 13,1 de la Ley vasca que afirma: "Corresponde al gobierno vasco, a través de la dirección de estadística, elaborar y tener al día el Censo Electoral».

Son dos los tipos de argumentos que, a mi entender, abonan la postura mantenida por el Estado, tanto en la impugnación de la Ley vasca como en la redacción de la estatal:

- En primer lugar, un argumento formal: en el Estatuto vasco no se menciona la competencia de elaboración del Censo Electoral. Dicha ausencia, que haría funcionar el mecanismo de atribución estatal de competencias residuales previsto en el artículo 149,3 de la Constitución ${ }^{28}$ no puede ser compensado ni por la competencia en legislación electoral, epígrafe bajo el que hay que entender las normas "centrales" del proceso electoral, ni tampoco por la competencia autonómica prevista en el artículo 37 del Estatuto: «Estadística del País Vasco para sus propios fines y competencias", ya que como ha declarado el Consejo de Estado, "la posible utilización del Censo para la formación de estadísticas no modifica el carácter del mismo como presupuesto de ejercicio del Derecho de sufragio y parte integrante del Régimen electoral general que impide que pueda ser regulado por la Comunidad Autónoma».

28 Vid sobre el articulo 149,3 mi trabajo en colaboración con el profesor J. GAAClA RocA, «Significado y alcance de la claúsula residual del artículo 149,3 de la Constitución", en Revista de política comparada, $.^{\circ} 7,1982$, págs. 167 y ss. Más recientemente he analizado el alcance que la Jurisprudencia del Tribunal Constitucional ha dado a dicha cláusula en el trabajo "Competencias estatutarias y jurisprudencia constitucional», en Revista de Estudios Políticos, n. ${ }^{\circ}$ 46-47 (Monográfico sobre el sistema político vasco), 1985, págs. 401-431, en especial 406 y ss. 
- En segundo lugar, un argumento material: la conexión del Censo con el Derecho de sufragio, en cuanto la inscripción en el mismo es uno de los requisitos fundamentales para su ejercicio, hace que, en estricta aplicación del artículo 149,1,1, el Censo deba ser configurado como un instrumento unitario, y por las especiales circunstancias de este documento, que consiste en un simple listado de ciudadanos con determinados datos y ordenados por Secciones y Mesas electorales, es materialmente imposible garantizar la igualdad si es confeccionado por dos autoridades administrativas distintas, aunque lo realicen sometiéndose a las mismas bases o principios. En este caso, la única forma real de garantizar un contenido unitario es siendo realizado por una única autoridad administrativa, que lógicamente ha de ser la estatal.

\section{A MODO DE CONCLUSION}

A tenor de todo lo expuesto, podemos concluir que la Ley Orgánica del Régimen Electoral General parece haber conseguido un adecuado equilibrio entre el inexcusable deber estatal de rodear al Derecho de sufragio activo y pasivo de todo un conjunto de instrumentos que garanticen su ejercicio en condiciones de igualdad en todo el territorio nacional, y la necesidad de un máximo respeto a las competencias de las Comunidades Autónomas, que les permita regular autónomamente los puntos esenciales de su propio sistema electoral, dentro del marco constitucional y estatutario.

Esta afirmación puede ser apoyada en dos datos:

- En primer lugar, en la aprobación prácticamente unánime de las Cámaras a la Ley Electoral, lográndose un clima de consenso desconocido en estos últimos años en las Cortes en torno a un tema tan fundamental, y, al mismo tiempo, de tan difícil acuerdo, por tratarse de una materia sumamente política.

- En segundo lugar, en el hecho de que las primeras Comunidades Autónomas que han elaborado sus propias leyes electorales, tras la aprobación de la LOREG, han adoptado prácticamente en su totalidad su contenido, incluso en temas en los que podrían haber actuado con absoluta autonomía.

Con ello es muy probable que la normativa electoral del conjunto de instituciones políticas del Estado termine por ser muy similar, lo que en modo alguno ha de ser visto como pérdida de autonomía, sino, por el contrario, como uso responsable de las facultades de autogobierno de las 
Comunidades Autónomas, y, en última instancia, como garantía de la funcionalidad del sistema democrático en su conjunto.

A pesar de este dato innegable, que en líneas generales hay que juzgar positivo, sin embargo, quizás se pueda echar en falta en la normativa regional hasta ahora aprobada, la introducción de elementos que, sin desvirtuar la necesaria igualdad, signifiquen un esfuerzo en la búsqueda de nuevas técnicas procedimentales que favorezcan el ejercicio del Derecho de sufragio y que, incluso, probada su eficacia, pudieran ser incorporadas al ordenamiento estatal. En este aspecto no han aprovechado, hasta ahora, la virtualidad de sus competencias electorales unida a la experiencia acumulada de procesos anteriores. Se ha preferido, por el contrario, seguir la senda, ciertamente más segura pero no necesariamente mejor, al menos en temas no fundamentales, de la imitación, cuando no la copia literal, en lugar de aportar nuevas formas al ejercicio del Derecho fundamental de sufragio. 